\title{
IMAGING GIGAHERTZ DYNAMICS IN MICROMECHANICAL RESONATORS USING ULTRAFAST PULSED LASER INTERFEROMETRY
}

\author{
L. Shao ${ }^{1,2}$, V.J. Gokhale ${ }^{1,3}$, J.C. Kuo ${ }^{4}$, A. Lal ${ }^{4}$, and J.J. Gorman ${ }^{1 *}$
}

${ }^{1}$ National Institute of Standards and Technology, Gaithersburg, Maryland, USA

${ }^{2}$ Department of Mechanical Engineering, University of Michigan, Ann Arbor, Michigan, USA

${ }^{3}$ Electrical Engineering and Computer Science Department, University of Michigan, Ann Arbor, Michigan, USA

${ }^{4}$ School of Electrical and Computer Engineering, Cornell University, Ithaca, New York, USA

\section{ABSTRACT}

Optical measurements of resonant MEMS can provide direct insight into their dynamics and guide improvements in device performance. This paper presents an optical method based on pulsed laser interferometry that can measure vibrations of MEMS resonators under coherent RF excitation with sub-picometer resolution and at arbitrary frequencies potentially well beyond 10 GHz. Vibration amplitude and phase can be measured across the entire resonator, thereby enabling experimental modal analysis and the imaging of complex dynamics, including mode mixing.

\section{INTRODUCTION}

Resonant MEMS operating at frequencies above $1 \mathrm{GHz}[1,2]$ are of great interest for efficient filtering and signal processing in wireless communications and advanced computing. However, the performance of these resonators is still not well understood, including their nonlinear dynamics, dissipation mechanisms, and vibrational modes. This is largely due to limitations in the measurement tools that are available. For example, electrical RF test equipment, although widely used, cannot provide information on mode shapes or the causes of acoustic loss. This paper presents a new interferometric approach that can measure vibrations beyond $10 \mathrm{GHz}$ with sub-picometer resolution, which provides direct insight into the dynamics of resonators operating above $1 \mathrm{GHz}$.

Conventional optical methods, including interferometric [3], photoelastic [4], and knife-edge techniques [4], use a continuouswave $(\mathrm{CW})$ laser whose frequency, phase, or intensity is modulated by resonator vibrations. For these methods, a photodetector with bandwidth greater than the vibration frequencies of interest must be used to measure the optical signal. However, resonator vibrations typically become progressively smaller with increasing frequency. Furthermore, the noise in photodetectors and amplifiers, parasitic capacitance and coupling in cables, and electromagnetic interference all increase with increasing frequency. The combination of these issues makes the use of conventional $\mathrm{CW}$ optical methods above $1 \mathrm{GHz}$ extremely challenging.

\section{INTERFEROMETRIC METHOD}

Due to the above limitations, we have developed a new approach using ultrafast pulsed laser interferometry (PLI) (Fig. 1(a)) that addresses these issues. The laser pulse train forms an electrical frequency comb with individual teeth equally spaced by the repetition rate $\left(f_{p}\right)$, as measured using a fast photodetector (Fig. 1(b)). By tuning $f_{p}$, one of the comb frequencies $\left(\mathrm{n} * f_{p}\right)$ can be placed close to the resonator excitation frequency $\left(f_{e x}\right)$, with a measurable offset $\left(f_{b}=\left|f_{e x}-\mathrm{n} f_{p}\right|\right)$. Thus, the laser pulse acts like a strobe that mixes down high-frequency vibrations to a beat signal, $f_{b}$. A lowbandwidth photodetector is used to measure $f_{b}$, which is processed with a lock-in amplifier. While stroboscopic interferometric techniques have been previously reported, they have been limited to operation either below $1 \mathrm{GHz}$ [5] or at discrete, widely separated frequencies [6]. Our approach works at any frequency above 0.95 $\mathrm{GHz}$ and has a noise floor comparable to a $\mathrm{CW}$ interferometer operating below $1 \mathrm{MHz}(\approx 30 \mathrm{fm} / \sqrt{\mathrm{Hz}}$, which is shot noise limited).

\section{RESULTS AND DISCUSSION}

Vibration results for two MEMS resonators are presented here. The first is a silicon width-extensional bulk acoustic resonator (BAR) [4] with a third-order in-plane mode around $0.983 \mathrm{GHz}$ (Fig. 2(a)). The out-of-plane displacement is measured, which results due to the Poisson effect. The excitation was swept by $1 \mathrm{MHz}$ around resonance, beating with the $19^{\text {th }}$ tooth $(981.35 \mathrm{MHz})$ of the comb $\left(f_{p}\right.$ $=51.65 \mathrm{MHz}$ ). The frequency response found with PLI is compared to that obtained by conventional CW laser interferometry in Fig. 3. The signal-to-noise ratio (SNR) is already $4 \mathrm{~dB}$ better for PLI while only just approaching $1 \mathrm{GHz}$. Figure 4 shows surface maps of the vibration amplitude $(\approx 3 \mathrm{pm})$ and phase for the third-order mode.

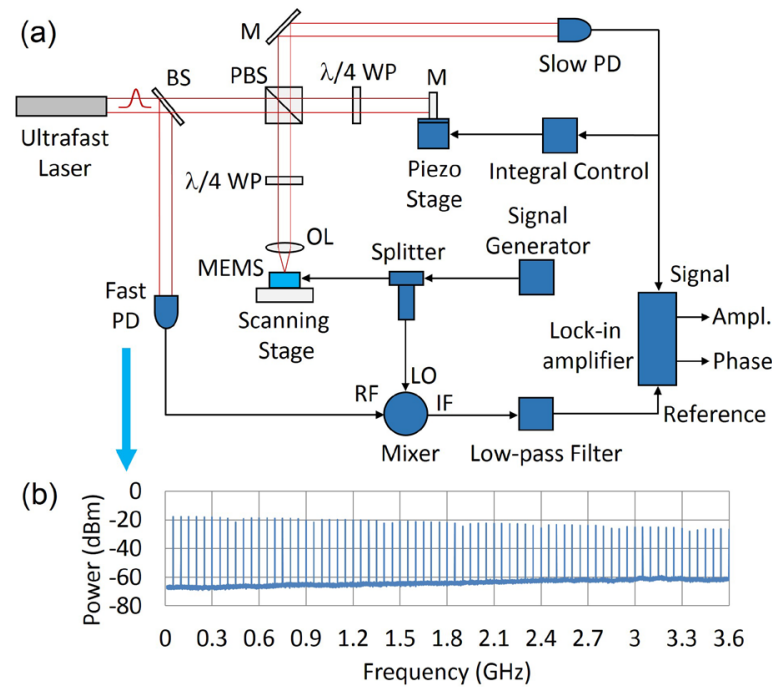

Figure 1: (a) Schematic of the ultrafast pulsed laser interferometer (PLI) (BS: beam splitter, PBS: polarizing beam splitter, PD: photodetector, M: mirror, $\lambda / 4$ WP: quarter wave plate, $O L$ : objective lens). (b) Power spectrum of the laser pulse train measured with a $12.5 \mathrm{GHz}$ fast photodetector, showing a frequency comb with individual teeth spaced by the pulse repetition rate (50.00 $\mathrm{MHz}$ ). The maximum operational frequency of the PLI is set by the bandwidth of the fast $P D$, the splitter, and the mixer.
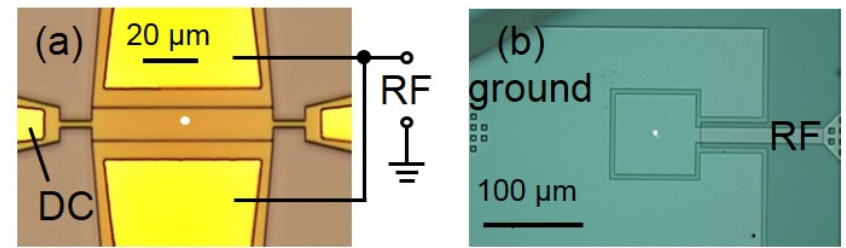

Figure 2: (a) Optical micrograph of a silicon width-extensional bulk acoustic resonator (BAR) with a width of $11.5 \mu \mathrm{m}$. Its measured third width-extensional mode is approximately $0.983 \mathrm{GHz}$. (b) Optical micrograph of an AlN bulk acoustic wave (BAW) transducer with a measured resonance frequency of approximately $2.35 \mathrm{GHz}$. The transducer size is $75 \mu \mathrm{m} \times 75 \mu \mathrm{m}$. Hilton Head Island, South Carolina, June 3-7, 2018 


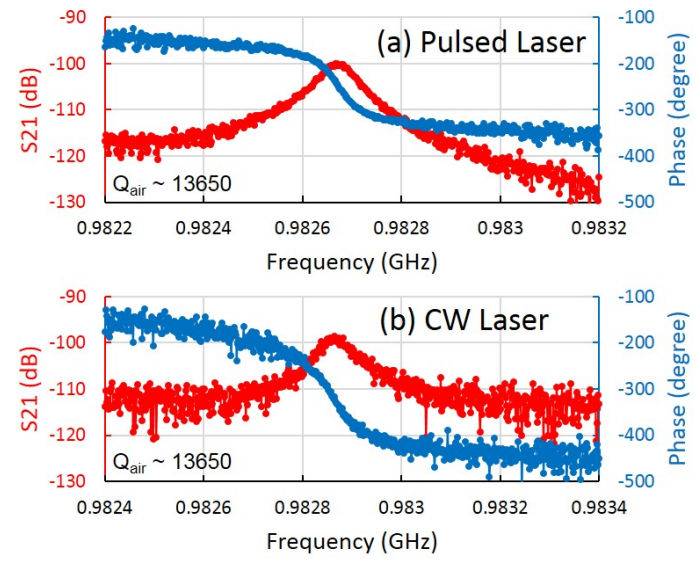

Figure 3: Frequency response of the BAR obtained by (a) pulsed laser interferometry and (b) $C W$ laser interferometry. The resonator was operated in air with $+10 \mathrm{dBm} R F$ power and $21 \mathrm{VDC}$ bias. The center frequencies differ slightly due to thermal drift and internal frequency inaccuracies in the different $R F$ instruments used.
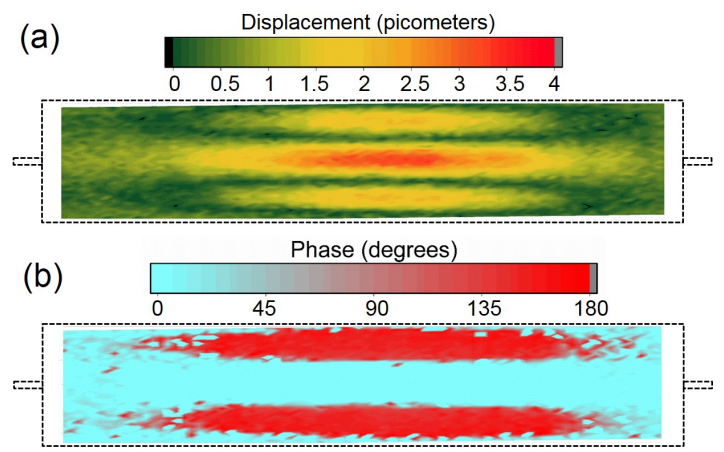

Figure 4: Mapping the BAR vibrations at $0.9827 \mathrm{GHz}$ using pulsed laser interferometry: (a) vertical vibration amplitude and (b) phase. Drive conditions: $21 \mathrm{VDC}$ bias and $+10 \mathrm{dBm} R F$ power. The dashed lines represent the outer dimensions of the BAR, $11.5 \mu \mathrm{m} \times$ $65 \mu \mathrm{m}$. The amplitude map clearly shows the expected third-order width-extensional mode. The phase transition along the width of the resonant body is nearly $180^{\circ}$, as expected.
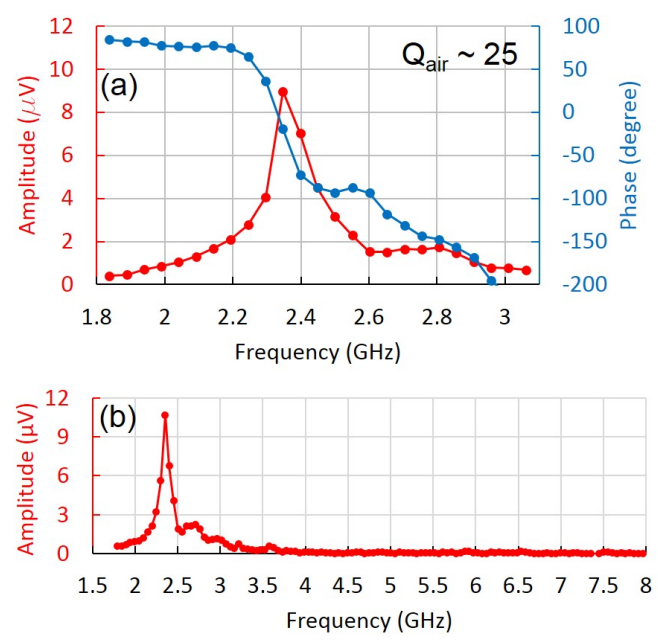

Figure 5: Frequency response of the AlN BAW obtained by pulsed laser interferometry, while operated in air with $+10 \mathrm{dBm} R F$ power: (a) mechanical resonance at $2.35 \mathrm{GHz}$ and (b) up to $8.0 \mathrm{GHz}$.

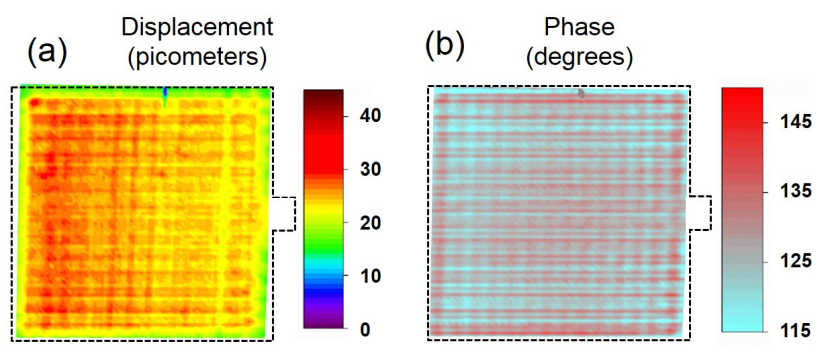

Figure 6: Mapping the BAW vibrations at $2.351 \mathrm{GHz}$ : (a) vibration amplitude and (b) phase. The dashed lines represent the perimeter of the BAW. The mode shape shows the superposition of the main thickness mode with other lateral resonance modes.

The second device is an AlN bulk acoustic wave transducer (Fig. 2(b)) [7]. Its fundamental mode at $2.35 \mathrm{GHz}$ is extremely difficult to measure using $\mathrm{CW}$ laser techniques. Since the resonance linewidth spans multiple comb teeth, the measurement is performed here by using a series of teeth $\left(f_{p}=50.0 \mathrm{MHz}\right)$ while $f_{b}$ is kept constant. The frequency response of the first thickness mode is shown to have an SNR of nearly $30 \mathrm{~dB}$ (Fig. 5(a)) and the BAW can be measured out to $8 \mathrm{GHz}$ (Fig. 5(b)). The vibration amplitude and phase maps (Fig. 6) show the superposition of multiple modes, demonstrating that PLI can reveal complex dynamics. Since both displacement and phase are obtained, animations of modes can be generated, making PLI an invaluable tool for investigating the dynamics of gigahertz resonators.

\section{ACKNOWLEDGMENTS}

Research performed in part in the NIST Center for Nanoscale Science and Technology Nanofab. BAW fabricated by A*STAR.

\section{REFERENCES}

[1] D. Weinstein, and S.A. Bhave, "The Resonant Body Transistor", Nano Letters, 10, 1234 (2010).

[2] M. Rinaldi, C. Zuniga, C. Zuo, and G. Piazza, "Super-highfrequency Two-port AlN Contour-mode Resonators for RF Applications", IEEE transactions on ultrasonics, ferroelectrics, and frequency control, 57, 38 (2010).

[3] K. Kokkonen, and M. Kaivola, "Scanning Heterodyne Laser Interferometer for Phase-sensitive Absolute-amplitude Measurements of Surface Vibrations", Applied Physics Letters, 92, 063502 (2008).

[4] V.J. Gokhale, and J.J. Gorman, "Approaching the Intrinsic Quality Factor Limit for Micromechanical Bulk Acoustic Resonators Using Phononic Crystal Tethers", Applied Physics Letters, 111, 013501 (2017).

[5] K.L. Telschow, V.A. Deason, D.L. Cottle, and J.D. Larson, "Full-field Imaging of Gigahertz Film Bulk Scoustic Resonator Motion", IEEE Transactions on Ultrasonics, Ferroelectrics, and Frequency Control, 50, 1279 (2003).

[6] T. Fujikura, O. Matsuda, D.M. Profunser, O.B. Wright, J. Masson, and S. Ballandras, "Real-time Imaging of Acoustic Waves on A Bulk Acoustic Resonator", Applied Physics Letters, 93, 261101 (2008).

[7] J.C. Kuo, J.T. Hoople, M. Abdelmejeed, M. Abdel-moneum, and A. Lal, "64-pixel Solid State CMOS Compatible Ultrasonic Fingerprint Reader”, 2017 IEEE 30 ${ }^{\text {th }}$ International Conference on Micro Electro Mechanical Systems (MEMS), Las Vegas, NV (2017), pp. 9-12.

\section{CONTACT}

*J.J. Gorman, tel: +1-301-975-3446; gorman@nist.gov 